\title{
Multi-phase postmortem CT angiography: recognizing technique-related artefacts and pitfalls
}

\author{
C. Bruguier • P. J. Mosimann • P. Vaucher • A. Uské • \\ F. Doenz • C. Jackowski $\cdot$ P. Mangin $\cdot$ S. Grabherr
}

Received: 15 August 2012 / Accepted: 25 February 2013 / Published online: 21 March 2013

(C) Springer-Verlag Berlin Heidelberg 2013

\begin{abstract}
Background and purpose Multi-phase postmortem CT angiography (MPMCTA) is increasingly being recognized as a valuable adjunct medicolegal tool to explore the vascular system. Adequate interpretation, however, requires knowledge about the most common technique-related artefacts. The purpose of this study was to identify and index the possible artefacts related to MPMCTA.

Material and methods An experienced radiologist blinded to all clinical and forensic data retrospectively reviewed 49 MPMCTAs. Each angiographic phase, i.e. arterial, venous and dynamic, was analysed separately to identify phasespecific artefacts based on location and aspect.

Results Incomplete contrast filling of the cerebral venous system was the most commonly encountered artefact, followed by contrast agent layering in the lumen of the thoracic aorta. Enhancement or so-called oedematization of the digestive system mucosa was also frequently observed.
\end{abstract}

C. Bruguier and P. J. Mosimann contributed equally to this work.

C. Bruguier $(\bowtie) \cdot$ P. Mangin $\cdot$ S. Grabherr

University Centre of Legal Medicine, Lausanne-Geneva,

University Hospital of Lausanne, Rue du Bugnon 21,

1011 Lausanne, Switzerland

e-mail: christine.bruguier@chuv.ch

C. Bruguier · P. J. Mosimann · A. Uské · F. Doenz

Department of Diagnostic and Interventional Radiology,

University Hospital of Lausanne, Rue du Bugnon 46,

1011 Lausanne, Switzerland

P. Vaucher

University Centre of Legal Medicine, Lausanne-Geneva,

University of Geneva, Rue Michel-Servet 1,

1211 Geneva 4, Switzerland

C. Jackowski

Center for Forensic Imaging, Institute of Forensic Medicine,

University of Bern, Buehlstr. 20,

3012 Bern, Switzerland
Conclusion All MPMCTA artefacts observed and described here are reproducible and easily identifiable. Knowledge about these artefacts is important to avoid misinterpreting them as pathological findings.

Keywords Postmortem CT · Postmortem angiography · Artefacts $\cdot$ Forensic radiology $\cdot$ Minimally invasive autopsy

\section{Introduction}

The evaluation of the human vascular system has always been a great challenge in postmortem investigations. First experiences using vascular casts enabling visualization of the vascular anatomy were performed in the seventeenth and eighteenth centuries by pioneers such as De Graaf, Ruysch, and Lower [1]. After the discovery of X-rays, postmortem angiography underwent a boom in the beginning of the twentieth century, during which hundreds of methods were developed to visualize the vascular system [2]. Especially the coronary arteries were investigated mostly using a technique described by Schlesinger [3] in 1938. Nevertheless, postmortem angiography had almost disappeared at the end of the twentieth century.

Nowadays, the status of postmortem angiography seems to be again in a state of change thanks to the increasing implementation of modern cross-sectional imaging techniques into postmortem investigations. The use of multidetector computed tomography in forensic medicine in particular is becoming more and more widespread [4-10]. Its introduction into forensic departments has added a rapid and easy method for collecting findings and conducting a digital documentation of the body. This new trend has naturally led to an increased interest in CT angiography. In fact, different research groups have already proposed new techniques [11] for performing CT angiography of the whole body [12-15] or of the coronary arteries only $[16,17]$. 
Recently, a new, standardized protocol for postmortem CT angiography has been developed that is easy to handle and reproducible and that permits a minimally invasive approach to studying blood vessels. This technique, called multiphase postmortem $\mathrm{CT}$ angiography (MPMCTA), consists of the acquisition of one native scan and three different phases of angiography: an arterial phase, a venous phase and a dynamic phase [18]. While the first two phases are performed to fill the arterial and the venous system to distinguish lesions in one system from the other, the dynamic phase is performed to confirm diagnosis.

Thanks to a complete filling of the vascular system of the head, thorax and abdomen, the images obtained by MPMCTA are of high quality. The performance of this exam allows the detection of haemorrhages, vessels rupture, stenosis, aneurysms and dissection even for small vessels [19-21]. This capability is unlike standard autopsy in which the search for these lesions is difficult and time-consuming or even impossible when small vessels are concerned. For this reason, the MPMCTA technique is used in our Institute of Legal Medicine routinely to contribute to the forensic expertise. For correct interpretation, two advanced radiologists (one vascular radiologist and one neuroradiologist) jointly evaluate all obtained images together with one forensic pathologist experienced in forensic imaging. However, in examining images from postmortem CT angiography, the different specialists have observed artefacts that seem to appear regularly in specific anatomical areas and that can induce misinterpretation. These artefacts differ from those typically observed on CTAs in the physiological conditions of a living body, where the heart and aortic pulsations as well diaphragmatic movements may induce motion artefacts and mimic aortic dissections or pulmonary embolism $[22,23]$. These observations lead us to ask ourselves the following questions: Does the perfusion of a dead body produce artefacts? Does the vascular system of a postmortem body react in the same way as the living body to contrast agent? And finally, do postmortem changes in the tissues generate artefacts?

Although many publications deal with the advantages and results of postmortem CT angiography, to our knowledge, none systematically describes the artefacts that can be observed. To interpret the obtained images, radiologists and forensic pathologists need to know what artefacts to anticipate. Because the MPMCTA technique was developed with the intention of applying it in routine investigations, knowledge about such pitfalls is highly important. The purpose of this study, therefore, was to identify and categorize MPMCTA artefacts according to their type, anatomical location and timing of appearance during the angiography.

\section{Material and methods}

Inclusion and exclusion criteria

We retrospectively included 54 consecutive MPMCTAs performed between January 2008 and September 2008 according to the standardized protocol proposed by Grabherr et al. in 2011 [18]. All MPMCTAs were performed before the conventional autopsy. Incomplete MPMCTAs or cases in which the order of the different phases was changed were excluded from the study. Subjects were included independent of the cause of death or medical history.

\section{Subjects}

Out of our 54 cases, five were excluded because of incomplete protocol implementations, such as a missing abdominal dynamic phase or inversion of the arterial and venous phases. In the remaining 49 subjects, there were 34 males and 15 females, median age and BMI were 52 years [1687 years] and 24.9 [17-36], respectively. MPMCTA was performed within $24 \mathrm{~h}$ after death in 36 cases, within 24 $48 \mathrm{~h}$ in seven cases, within $48-72 \mathrm{~h}$ in four cases and after more than $72 \mathrm{~h}$ in two cases. There were 27 natural deaths (such as sudden cardiac arrest), 13 traumatic deaths (such as from motor vehicle accidents) and nine deaths due to intoxication (recreational or prescribed drugs). All data about the examined cases are provided in Table 1.

\section{Postmortem examinations}

A native $\mathrm{CT}$ scan was performed prior to any manipulation of the corpse using an eight-row CT unit (CT LightSpeed 8, GE Healthcare, Milwaukee, WI, USA) with scan parameters as given in Table 2 covering the brain, the neck, the chest and the abdomen to the ischium. CT-guided sampling was then performed by trained forensic radiographers [18, 24] and included bilateral needle lung biopsies and puncture of the right cardiac cavities, gallbladder and bladder to sample cardiac blood, bile and urine, respectively.

To subsequently perform MPMCTA, unilateral cannulation of the femoral vessels was achieved using 16- or 18-Fr cannulae (MAQUET Gmbh \& Co. KG, Rastatt, Germany) for the artery or vein, respectively. A recently developed pressure-controlled perfusion device (Virtangio ${ }^{\circledR}$, Fumedica AG, Muri, Switzerland) was used to inject a mixture of contrast agent (Angiofil ${ }^{\circledR}$, Fumedica AG, Muri, Switzerland) with paraffin oil (paraffinum liquidum, obtained in local pharmacy). According to the protocol proposed by Grabherr et al. [18], the arterial phase of angiography was then carried out after having injected 1,200 ml contrast-agent mixture $\left(6 \%\right.$ Angiofil $\left.^{\circledR}\right)$ retrograde during $90 \mathrm{~s}$ into the arterial system. For the venous phase of 


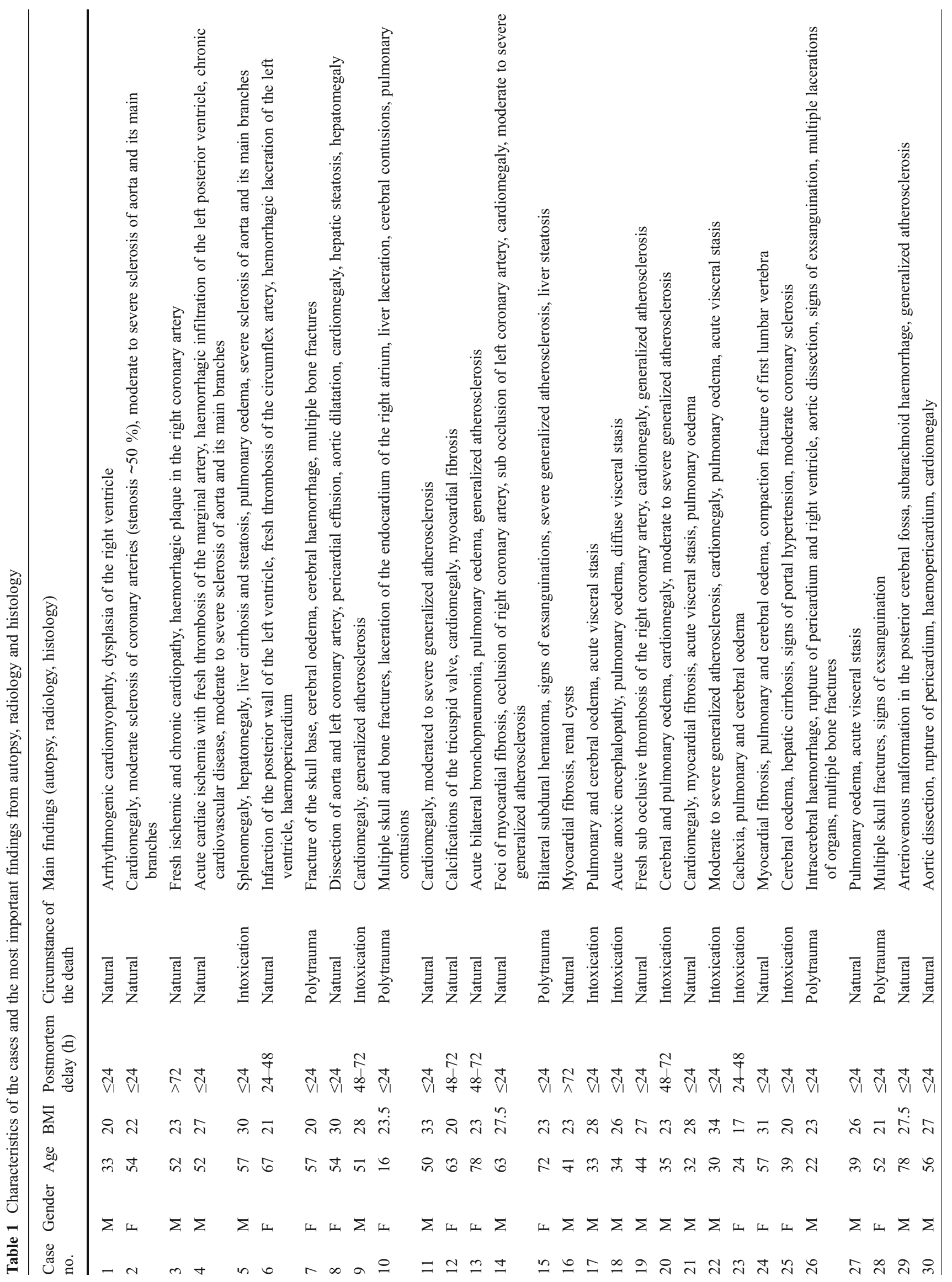




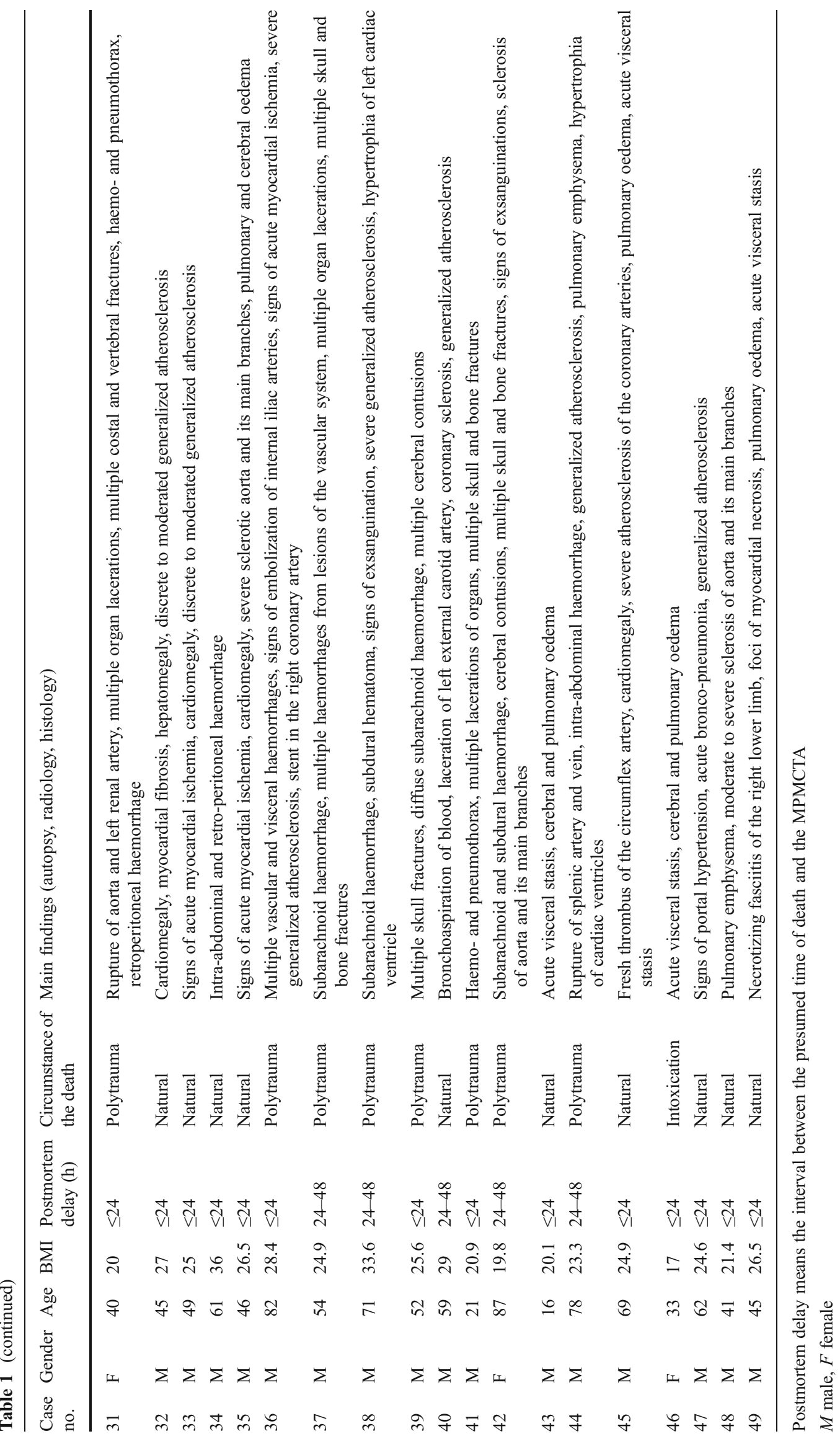


Table 2 Scan parameters used for acquisition of the radiological images (for native CT scans and the different angiographic phases)

\begin{tabular}{llllllll}
\hline & $\begin{array}{l}\text { Field of view } \\
(\mathrm{cm})\end{array}$ & $\begin{array}{l}\text { Slice } \\
\text { thickness }\end{array}$ & $\begin{array}{l}\text { Reconstruction interval } \\
(\mathrm{mm})\end{array}$ & $\begin{array}{l}\text { Tube voltage } \\
(\mathrm{kV})\end{array}$ & $\begin{array}{l}\text { Milliamperes } \\
\text { (Ma modulated) }\end{array}$ & Tube rotation (s) & Pitch \\
\hline Native & 50 & 1.25 & 1 & 120 & 280 & 0.8 & 0.875 \\
Arterial & 50 & 1.25 & 0.6 & 120 & 280 & 0.8 & 0.875 \\
Venous & 50 & 2.5 & 2 & 120 & 280 & 0.875 \\
Dynamic & 50 & 2.5 & 2 & 120 & 280 & 0.8 & 0.875 \\
\hline
\end{tabular}

angiography, $1,800 \mathrm{ml}$ of contrast-agent mixture was injected antegrade into the femoral vein during $135 \mathrm{~s}$. At least, the dynamic phase of MPMCTA was carried out by injecting further $500 \mathrm{ml}$ of contrast-agent mixture into the femoral artery and performing the radiological data acquisition during an ongoing perfusion with $3 \mathrm{ml} / \mathrm{s}$ during $150 \mathrm{~s}$. The used scan parameters for angiography are given in Table 2 . The data acquisition during the angiography covered the same anatomical regions as the native CT scan (head, neck, thorax and abdomen).

For each exam, a board-certified forensic pathologist with experience in forensic imaging and two boardcertified radiologists specialized in vascular- and neuroradiology established a detailed radiological report describing all findings in the different phases. These reports are edited as a routine in our centre in order to complete the autopsy reports. Two forensic pathologists, one board-certified and one in training, subsequently carried out a conventional autopsy in accordance with international guidelines $[25,26]$, which was documented in a detailed autopsy report edited by the two physicians.

\section{Data collection}

Based on more than 150 MPMCTAs interpreted at our institution over the last 2 years, we established a list of all encountered artefacts that have been described in the routine radiological reports. According to our experience, they typically occur in the digestive and vascular systems, as well as at biopsy and puncture sites. With this knowledge, we distinguished two types of artefacts according to the anatomical area in which they accrued. The first area was the vascular system of the neck and the brain, and the second area included the chest and the abdomen.

The data of the first area could be extracted from the routine radiological reports edited by two radiologists and one forensic pathologist. The data of the second area were not available in the radiological report and were therefore assembled by a board-certified radiologist who was blinded to the results of the previous radiological reports, the results of the autopsies and all case information.
First anatomical area: vascular system of the neck and the brain Our experiences showed that artefacts in this anatomical area are visible as incomplete perfusion of the vessels, especially the venous system of the head. In order to evaluate the perfusion of the vessels of the neck and the brain, we investigated the appearance of these artefacts. As incomplete fillings of the vessels are documented in the routine radiological report, we extracted these data retrospectively. To quantify the degree of contrast perfusion of the cerebral venous system, we developed a nine-point (from 0 to 8 ) scaling system, as shown in Fig. 1.

Second anatomical area: chest and the abdomen In order to investigate the prevalence of artefacts in the chest and abdomen, we based our study on the experiences we had. A total of 28 sites in which artefacts have been described during the routine radiological interpretation of the last years have been selected. In order to allow an evaluation

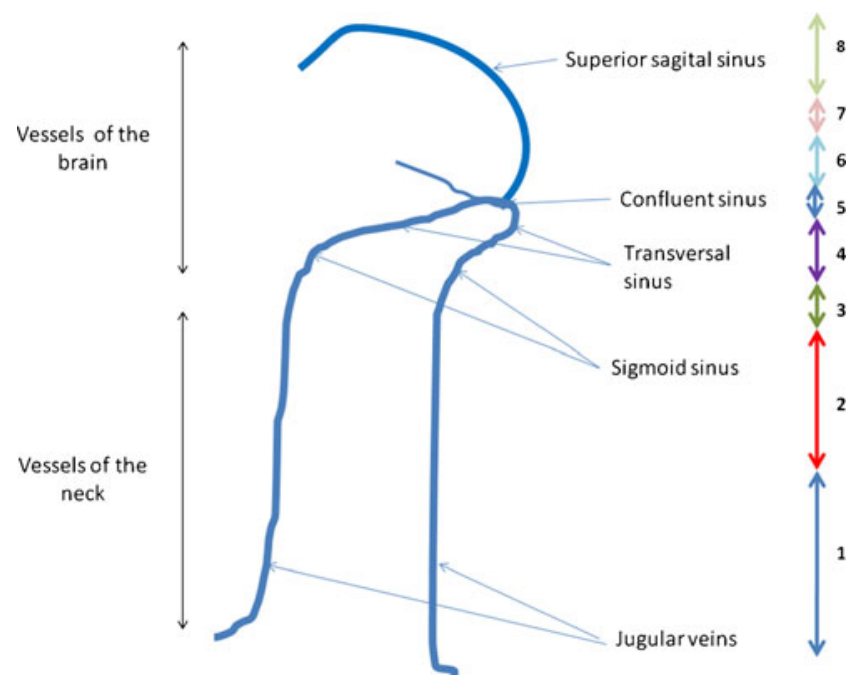

Fig. 1 Degree of retrograde contrast filling of the head and neck venous system: 0: no opacification of the precerebral and cerebral veins, 1 partial opacification of the jugular veins, 2 full opacification of the jugular veins, 3 opacification of the sigmoid sinus, 4 opacification of the transverse sinus, 5 opacification of the torcular, 6 opacification of $1 / 3$ of the superior sagittal sinus, 7 opacification of $2 / 3$ of the superior sagittal sinus and 8 full opacification of superior sagittal sinus (corresponding to a complete opacification of the cerebral venous system) 
in a systematic fashion, these 28 sites have been entered into an Excel spreadsheet. Each artefact was specifically defined by the board-certified forensic pathologist and radiologist used to investigate the radiological data and explained to the blinded radiologist in charge of reviewing the exams (see Table 3). This radiologist evaluated each site in all three angiographic phases. Artefacts were coded as 0,1 or 2, respectively, if they were present, absent or impossible to evaluate (e.g. the region of interest was outside the scanned field of view). Space was given for free comments, such as the identification of a new artefact. The defined artefacts were observed in the digestive system, the vascular system and at sampling sites.

\section{Digestive system}

Artefacts of the gastrointestinal mucosa were graded as follows: (1) mucosal enhancement only (normal thickness), (2) oedematous enhancement (thickened mucosa) and (3) contrast agent extravasation into the digestive lumen (Fig. 2a).
Peripancreatic contrast extravasation was also evaluated as a separate artefact (Fig. 2b).

\section{Vascular system}

The quality of the perfusion was determined by the degree of contrast filling on each angiographic phase, i.e. full, partial or no vessel opacification (Fig. 3a). Layering of the contrast agent, as characterized by a denser layer of contrast above the more dependent stagnating and hypodense blood (Fig. 3b), was the most prominent vascular artefact in our early experience. We thus predefined a number of target vessels where this seemed to occur the most. In the arterial phase, we examined the left cardiac cavities, the ascending and descending aorta, the aortic arch, the coronary arteries and the pulmonary veins. Sites of interest in the venous phase were the right cardiac cavities, the pulmonary arteries, the superior and inferior vena cava, the portal vein and the renal veins. In the dynamic phase, all of the aforementioned arterial and venous sites were re-evaluated.

Table 3 List and definition of artefacts

\begin{tabular}{|c|c|c|c|c|c|}
\hline \multirow[t]{2}{*}{ Artefact } & \multirow[t]{2}{*}{ Definition } & \multirow[t]{2}{*}{ Localization } & \multicolumn{3}{|c|}{ Phase of angiography } \\
\hline & & & Arterial & Venous & Dynamic \\
\hline \multirow[t]{3}{*}{ Filling defects } & \multirow[t]{3}{*}{$\begin{array}{l}\text { Inhomogeneous opacification without } \\
\text { layering }\end{array}$} & $\begin{array}{l}\text { Ascending aorta-aortic arch- } \\
\text { descending aorta-coronary arteries-- } \\
\text { pulmonary veins }\end{array}$ & $\mathrm{X}$ & & $\mathrm{X}$ \\
\hline & & $\begin{array}{l}\text { Left atrium-left ventricle-right } \\
\text { atrium-right ventricle }\end{array}$ & $\mathrm{X}$ & $\mathrm{X}$ & $\mathrm{X}$ \\
\hline & & $\begin{array}{l}\text { Pulmonary arteries-superior vena } \\
\text { cava-inferior vena cava portal veins }\end{array}$ & & $\mathrm{X}$ & $\mathrm{X}$ \\
\hline \multirow[t]{3}{*}{ Layering } & \multirow[t]{3}{*}{$\begin{array}{l}\text { Hyperdense layer of contrast above } \\
\text { stagnating and hypodense blood }\end{array}$} & $\begin{array}{l}\text { Ascending aorta-aortic arch- } \\
\text { descending aorta-coronary arteries-- } \\
\text { pulmonary veins }\end{array}$ & $\mathrm{X}$ & $\mathrm{X}$ & $\mathrm{X}$ \\
\hline & & $\begin{array}{l}\text { Left atrium-left ventricle-right } \\
\text { atrium-right ventricle }\end{array}$ & $\mathrm{X}$ & $\mathrm{X}$ & $\mathrm{X}$ \\
\hline & & $\begin{array}{l}\text { Pulmonary arteries-superior vena } \\
\text { cava-inferior vena cava portal veins }\end{array}$ & & $\mathrm{X}$ & $\mathrm{X}$ \\
\hline $\begin{array}{l}\text { Peripancreatic contrast } \\
\text { extravasation }\end{array}$ & $\begin{array}{l}\text { Visualization of contrast agent around } \\
\text { the pancreas }\end{array}$ & Pancreas & $\mathrm{X}$ & $\mathrm{X}$ & $\mathrm{X}$ \\
\hline $\begin{array}{l}\text { Extravasation of contrast } \\
\text { from renal vein }\end{array}$ & $\begin{array}{l}\text { Visualization of contrast around the } \\
\text { renal vein }\end{array}$ & Right renal vein-left renal vein & & $\mathrm{X}$ & $\mathrm{X}$ \\
\hline $\begin{array}{l}\text { Mucosal enhancement } \\
\text { (digestive system) }\end{array}$ & $\begin{array}{l}\text { Enhancement of the mucosa without } \\
\text { increase of thickness }\end{array}$ & Stomach-duodenum-colon & $\mathrm{X}$ & $\mathrm{X}$ & $\mathrm{X}$ \\
\hline $\begin{array}{l}\text { Oedematous enhancement } \\
\text { of mucosa (digestive } \\
\text { system) }\end{array}$ & $\begin{array}{l}\text { Enhancement of the mucosa with } \\
\text { increasing thickness }(\geq 0.5 \mathrm{~cm})\end{array}$ & Stomach-duodenum-colon & $\mathrm{X}$ & $\mathrm{X}$ & $\mathrm{X}$ \\
\hline $\begin{array}{l}\text { Intraluminal extravasation } \\
\text { (digestive system) }\end{array}$ & $\begin{array}{l}\text { Extravasation of contrast agent into the } \\
\text { lumen of the digestive system }\end{array}$ & Stomach-duodenum-colon & $\mathrm{X}$ & $\mathrm{X}$ & $\mathrm{X}$ \\
\hline $\begin{array}{l}\text { Artefacts related to } \\
\text { sampling }\end{array}$ & $\begin{array}{l}\text { Extravasation of contrast resulting from } \\
\text { the passage of the biopsy or puncture } \\
\text { needle }\end{array}$ & Heart-lungs-gallbladder-bladder & $\mathrm{X}$ & $\mathrm{X}$ & $\mathrm{X}$ \\
\hline Other artefacts & $\begin{array}{l}\text { All other non-defined artefacts } \\
\text { observed }\end{array}$ & - & $\mathrm{X}$ & $\mathrm{X}$ & $\mathrm{X}$ \\
\hline
\end{tabular}

$X$ evaluated in this phase of angiography 

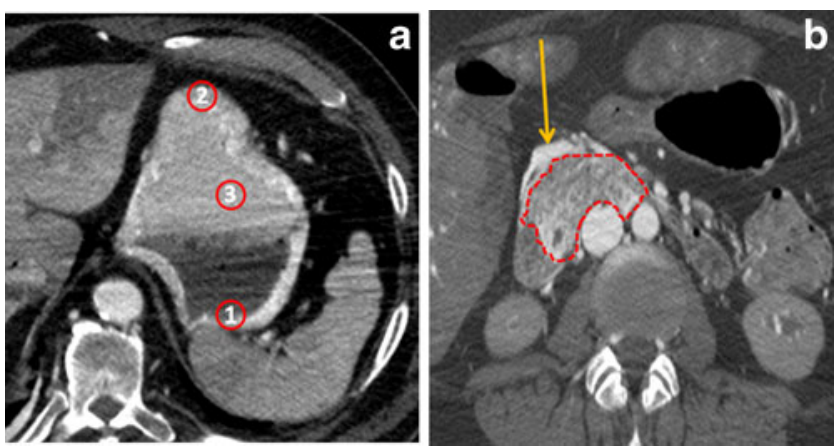

Fig. 2 Artefacts in the digestive system a: Axial images showing the stomach and the 3 predefined artefacts: 1: Enhancement of the gastric mucosa. 2: Enhancement and oedema of the gastric mucosa. 3: Enhancement and oedema of the gastric mucosa with extravasation of contrast agent into the gastric lumen. b: Axial image showing the pancreatic head (red dotted) encircled with an artefactual extravasation of contrast agent (orange arrow)

\section{Artefacts related to sampling}

Because samples are collected prior to the injection of contrast agent into the vascular system, the passage of the needle understandably can create artefacts resulting from lesions of small vessels (Fig. 4). The second radiological view performed by the observer should therefore indicate whether an artefact was visible at each puncture site or not.

\section{Evaluation of the relationship between case information and} the presence of artefacts To verify whether certain parameters influenced the presence or the amount of artefacts, the following data were extracted from the autopsy reports: age, sex and BMI of the individual; presumed postmortem interval between the death and the MPMCTA; and circumstance of death (Table 1). To simplify our statistical analyses, circumstances of death were divided into polytrauma, sudden death from natural or unknown causes and intoxication, designated as groups 1, 2 and 3, respectively.

Verification of the presence of artefacts In order to verify that the described artefacts were really artefacts and no pathological findings, all data have been correlated to the autopsy results by viewing the autopsy reports and cross checking the findings described there with the artefacts described by the different radiologists.

\section{Statistical analyses}

Arithmetic means were calculated for quantitative values. We used non-parametric tests (Wilcoxon rank-sum test for quantitative variables, Fisher's exact test for qualitative variables or Spearman's rho correlation coefficient) to test correlations and associations between case information and presence of artefacts. Significance level was set at $p<0.05$ without adjustment for multiple testing.

\section{Results}

Neck and brain perfusion

In the arterial phase, no incomplete perfusion of the precerebral and intracerebral arteries was observed. In the venous phase, however, the venous system was completely opacified in only $20.4 \%$ of cases. In the dynamic phase, this number slightly increased to $22.4 \%$ (Fig. 5); this means we observed an improvement of the filling of the venous system in four cases in the dynamic phase compared to the venous phase. The only cases in which complete venous perfusion could be observed were those who died due to a polytrauma (Table 4).

Evaluation of gastrointestinal artefacts

In the digestive system, artefacts could be observed regularly (Fig. 6). Their prevalence increased slightly with an increasing number of effected phases. The upper gastrointestinal tract was more often affected than the lower. Artefact prevalence was higher in the stomach, followed by the duodenum, and artefacts were rarely observed in the large bowel. Grade 1 artefacts (enhancement of the mucosa) were more often observed than grade 2 artefacts (oedematous enhancement of the mucosa). Therefore, after the arterial phase, an artefact grade 1 could be observed in $81.6 \%$ of the cases in the stomach, in $65.3 \%$ in the duodenum and $59.2 \%$ in the colon. During the following phases, its incidence did only increase slightly (Fig. 6a). After the arterial phase, grade 2 artefacts were observed in $51 \%$ of the cases in the stomach, in $22.4 \%$ in the duodenum and in $4.1 \%$ in the colon. Their incidence increased slightly during the venous phase, especially in the colon (6.1\%) (Fig. 6b). Grade 3 artefacts (oedematous enhancement of the mucosa with contrast extravasation into the
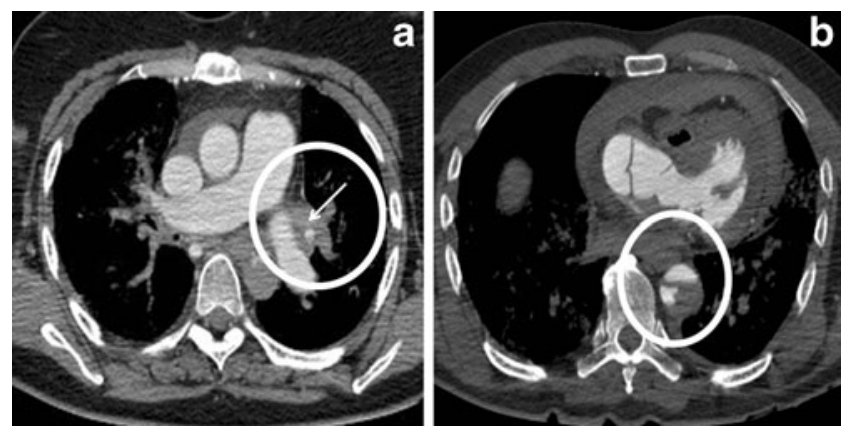

Fig. 3 Artefacts in the vascular system. a Perfusion defect of the pulmonary arteries (white arrow in the white circle). b Layer mimicking a dissection of the abdominal aorta (white circle) 

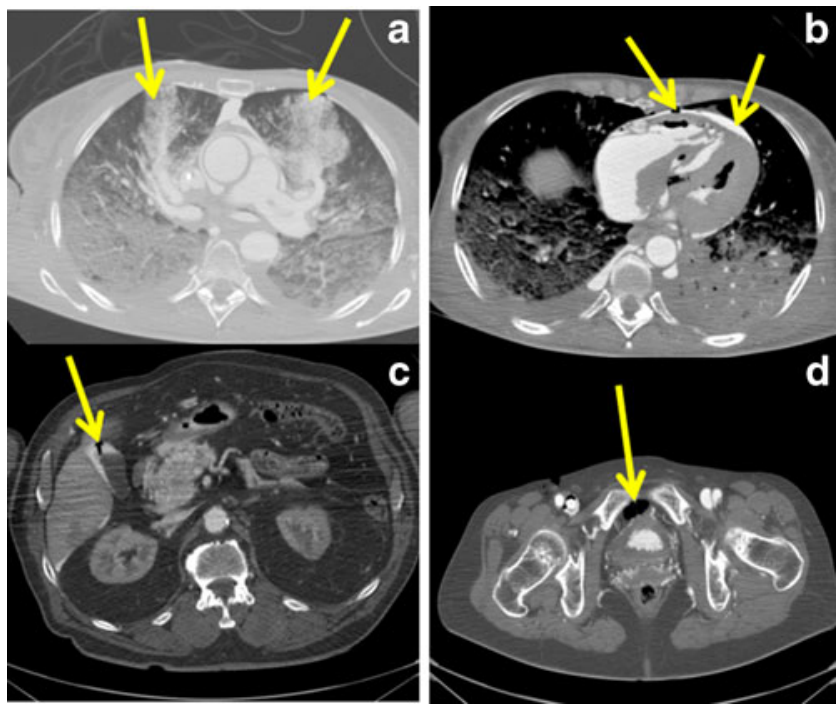

Fig. 4 Artefacts resulting from sample collection. a Diffuse contrastagent extravasations in the trajectory of the biopsy needles visible in both lungs (yellow arrow). b Presence of gas in the trajectory of the needle and contrast agent in pericardium (yellow arrow) resulting from the aspiration of cardiac blood. c Contrast-agent extravasation and presence of gas (yellow arrow) and around the gallbladder resulting from the passage of the needle inserted for bile aspiration. $\mathbf{d}$ Presence of gas (yellow arrow) in the trajectory of the needle resulting from collection of urine

digestive lumen) appeared more often in later phases of angiography, mostly in the venous phase (in $28.6 \%$ of the cases in the stomach and in $6.1 \%$ in the colon) or in the dynamic phase (in $10.2 \%$ of the cases in the duodenum). Modest peripancreatic contrast extravasation was observed mostly during the arterial phase (in $46.9 \%$ of the cases) and more rarely during the venous phase (Fig. 6c).
Artefacts of the vascular system

\section{Contrast agent layering}

Contrast agent layering was observed in all of the predefined vessels (Fig. 7), proportionally more so in larger arteries and veins (in $67.3 \%$ of the cases in the descending aorta during the arterial phase and the inferior vena cava during the venous phase). It was also frequent in the cardiac cavities (in $40.8 \%$ of the cases in the left atrium; in $55.1 \%$ in the right atrium; in $16.3 \%$ in the right ventricule; in $36.7 \%$ in the left ventricule during the venous phase). In the circulating phase, the prevalence of contrast layering increased in most vascular compartments except for some sites where the contrast layer decreased and sometimes even disappeared. These sites were the ascending aorta (minus $18.3 \%$ ), the portal vein (minus $4.1 \%$ ) and the left cardiac ventricle (minus $4 \%$ ). Contrast layering in the coronary arteries and right atrium remained stable (unchanged) in all three angiographic phases $(6.1 \%$ for the coronary arteries and $55.1 \%$ for the right atrium).

\section{Degree of vessel opacification}

Filling defects or inhomogeneous opacification without layering (Fig. 8) was essentially observed in the cardiac cavities. Prevalence reached $85 \%$ for the left atrium, $53.1 \%$ for the left ventricle and $77.6 \%$ for both the right cavities (atrium and ventricle) in the arterial phase. Inhomogeneous opacification was also observed in the pulmonary arteries in $83.7 \%$ of cases during the arterial phase. However, the
Fig. 5 Evaluation of the perfusion of neck and brain. The different degrees of perfusion are shown in different colours for the venous and for the dynamic phase according to the colour scheme shown in Fig. 1: from blue colour for no opacification of the vessels ( 0 ) to green for a complete opacification of the cerebral venous system (8). The numbers in the figure correspond to the number of cases which have obtained the corresponding degree

\section{Perfusion of the venous system of the head}

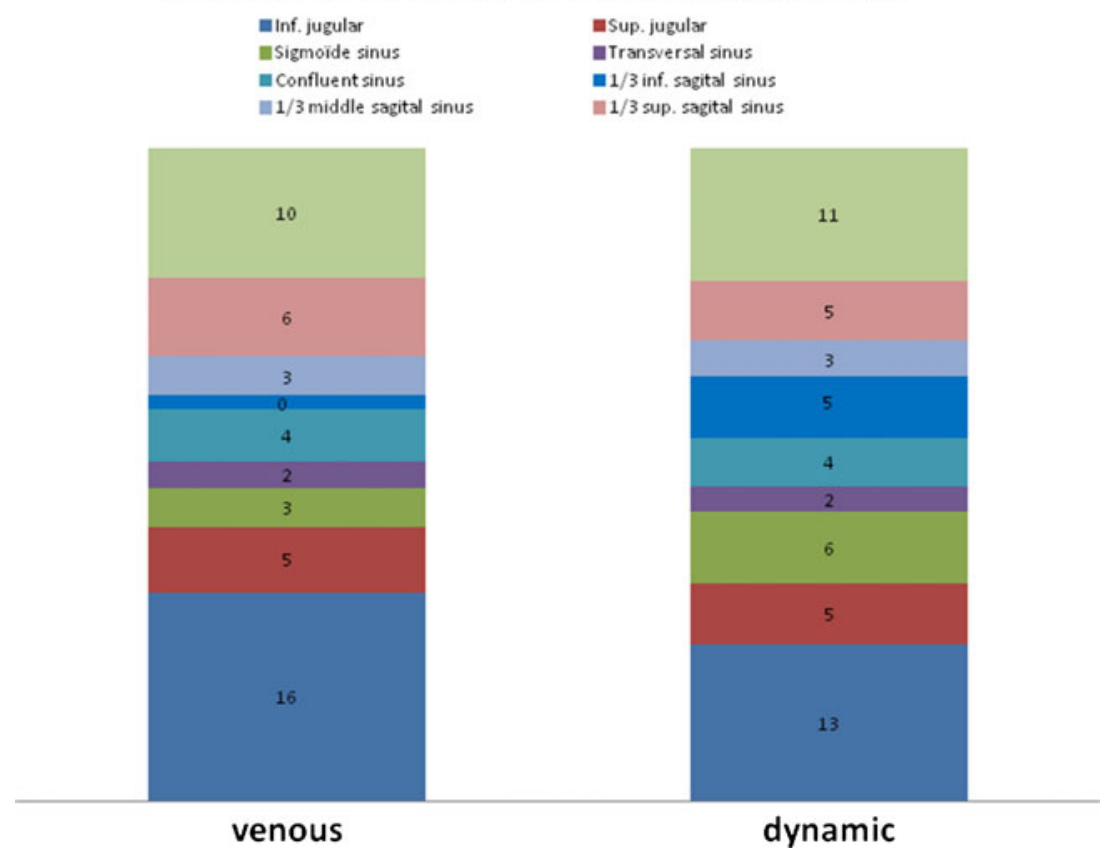




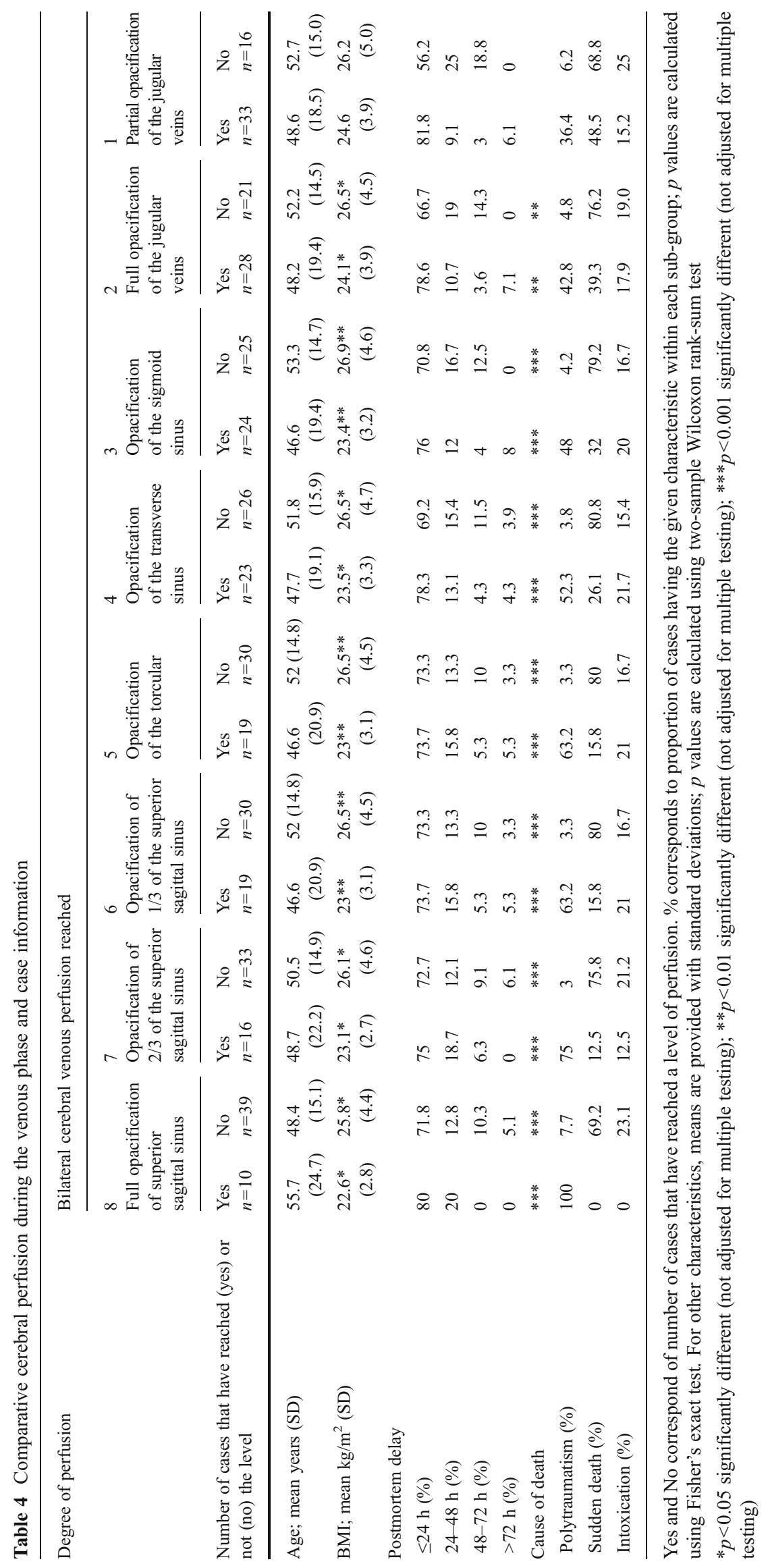




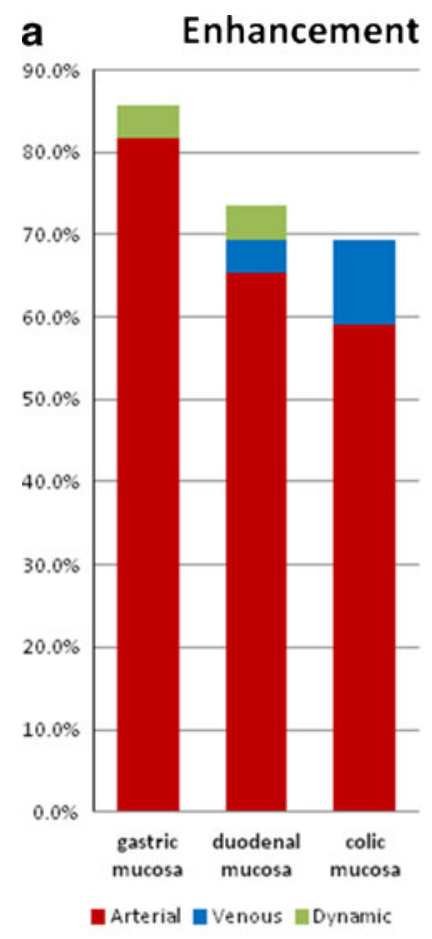

b

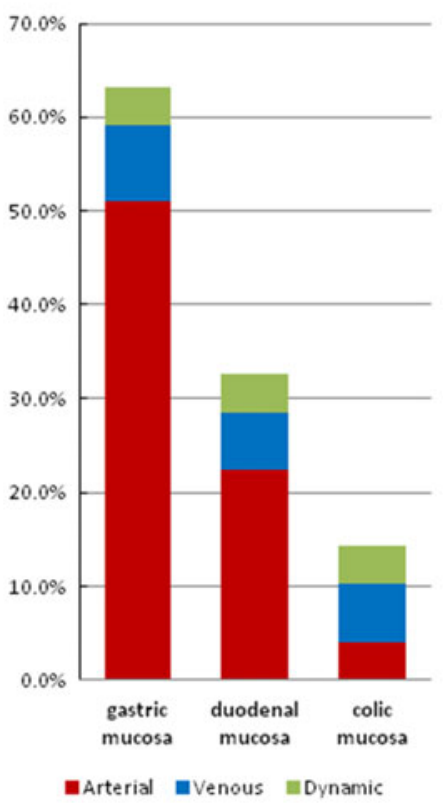

C Extravasation

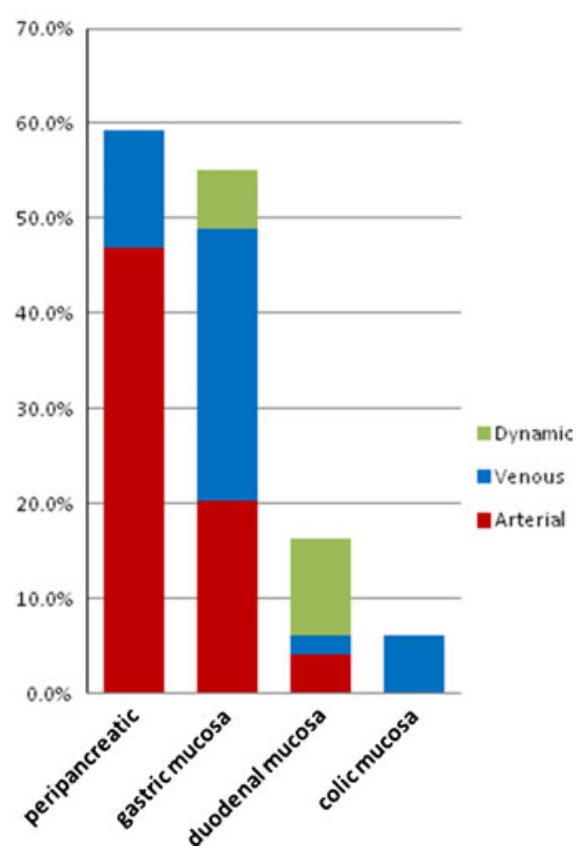

Fig. 6 Prevalence of gastrointestinal artefacts. a Diagram of the prevalence of an enhancement of the digestive mucosa, which was most frequently observed in the gastric mucosa $(81.6 \%)$, followed by the duodenal mucosa $(65.3 \%)$ and the colic mucosa $(59.2 \%)$ in the arterial phase. The prevalence of this artefact increased slightly between the other phases. b Diagram of the prevalence of mucous oedema, which shows the same distribution in the arterial phase with smaller values than that of a simple enhancement: $51 \%$ for the gastric mucosa, $22.4 \%$ for the duodenal mucosa and $4.1 \%$ for colon mucosa. A slight increase

prevalence of this phenomenon decreased significantly with the increasing number of performed angiographic phases. For example in the pulmonary arteries, such artefacts remained visible only in $4.1 \%$ of the cases.

Artefacts caused by CT-guided fluid and tissue sampling

Artefacts related to the biopsy or needle tracts were not frequently observed in the arterial phase (Fig. 9). In the venous phase, however, their cumulative prevalence significantly increased from 12.2 to $79.5 \%$ for the right lung, from 8.2 to $59.2 \%$ for the left lung, from 6.1 to $67.3 \%$ for the gallbladder and from 24.5 to $69.4 \%$ for the right ventricle. The least affected sampled site was the bladder, where artefacts were never observed in the arterial phase. They were present in $10.2 \%$ of cases in the venous phase, however.

Other artefacts

During the venous and circulating phases, contrast extravasation from the right renal vein was observed in seven cases $(14.3 \%)$. of the prevalence can be observed after the venous and dynamic phase. c Diagram of the prevalence of intraluminal contrast-agent extravasation and extravasation surrounding the pancreatic head. In the arterial phase, this artefact could be observed in $20.4 \%$ in the stomach and in $4.1 \%$ in the duodenum but was never observed in the colic lumen. Again, an increase in the prevalence could be observed after the different phases, especially the venous phase. The prevalence of a peripancreatic extravasation could be calculated as $46.9 \%$ after the arterial phase, with a slight increase during the venous phases $(12.2 \%)$

Associations between artefacts and case information

Cause of death and BMI influenced the degree of opacification of the neck and brain venous system (Table 4). Complete perfusion was more frequently observed with lower BMI ( 22.5 vs. $25.8 ; p=0.033)$ and in polytrauma cases (100 vs. $5.3 \% ; p<0.001)$. Likewise, a higher BMI was more frequently associated with artefacts (Spearman' rho $=0.300 ; p=0.0366$ ), as opposed to polytrauma cases, in whom artefacts were less often observed (3.2 vs. $5.2 ; p=0.004)$.

We did not find any correlation between the presence of artefacts and age (Spearman's rho $=0.138 ; p=0.345$ ) or time since death (Spearman's rho $=0.174 ; p=0.232$ ). However, we noted that cases with reduced cerebral perfusion presented also more artefacts in the second anatomical area including thorax and abdomen (Spearman's rho $=-0.396 ; p=0.005$ ).

Verification of the presence of artefacts

By comparing the described artefacts with the autopsy reports, we could clearly identify those as artefacts and rule out a misinterpretation of real findings (e.g. exclusion of 
Fig. 7 Prevalence of contrast layering
Prevalence of a layer in the vascular system

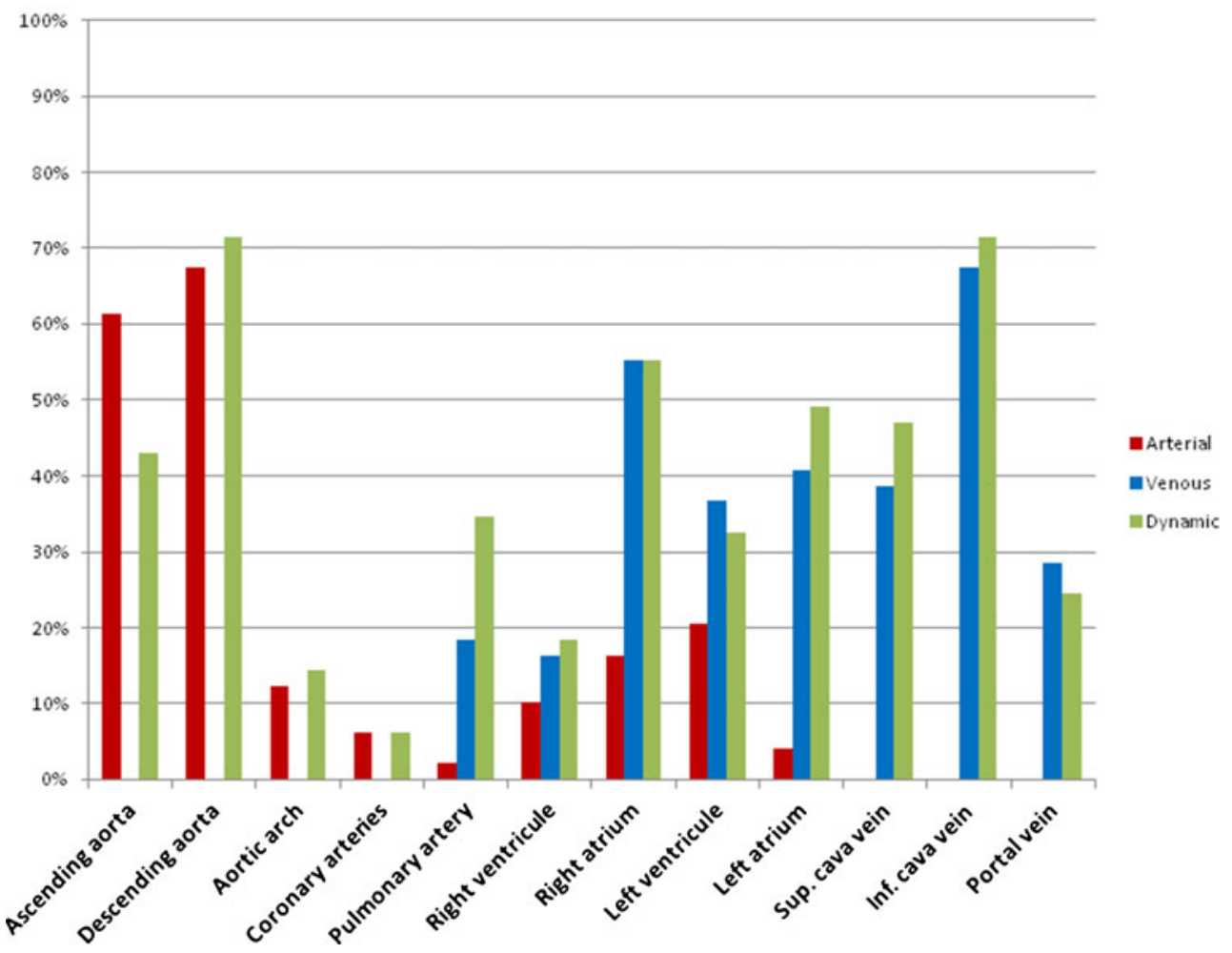

pulmonary embolism when an inhomogeneous opacification of the pulmonary arteries has been described). In our study, there was no case in which a pathological finding has been interpreted as artefact.

\section{Discussion}

Our study evaluated and described the artefacts that can be observed during interpretation of radiological images obtained from postmortem CT angiography using MPMCTA. Although MPMCTA aims at mimicking in vivo physiology, the perfusion conditions are reversed because the arterial system is opacified in a retrograde fashion. Also postmortem changes can influence the obtained radiological images. Such conditions may favour the appearance of artefacts that differ from those observed under normal physiologic conditions in life when the beating heart distributes contrast peripherally.

In our department, forensic pathologists have been performing joint interpretations with radiologists for the last 3 years. Based on our preliminary experience in more than 150 cases, we have noticed artefacts which have been observed regularly. We could group three types of such technique-related artefacts or pitfalls that may mimic pathologic findings: (1) incomplete venous opacification of the head and neck vessels, (2) artefactual contrast enhancement or extravasation of the gastrointestinal tract and (3) contrast layering in the non-dependent aspect of vessels or incomplete filling of the arterial or venous system. To systematically evaluate their prevalence, we performed this study.

Concerning neck and brain perfusion, our systematic evaluation of 49 consecutive MPMCTAs could show that the arteries of the head and neck were always fully opacified, independent of BMI or the postmortem delay. A complete filling of the cerebral venous system, however, was rarely observed. The reason the venous system is more difficult to perfuse probably lies in the structural differences between the wall composition of arteries and veins. Arteries have a thicker muscular layer, allowing them to tolerate higher pressures and to be more resistant to extravasation than veins, which may explain why the arteries are so well opacified. Conversely, veins have a thinner muscular layer and are less resistant to higher pressures, despite their capacity to contain larger volumes. Of interest, the venous system was more often fully perfused when trauma was the cause of death. Because polytrauma victims typically die in acute shock within seconds or minutes, it is possible that vasoconstrictive factors may influence the antemortem state of the venous system. In contrast to arteries, BMI influenced the degree of venous filling, and adapting the volume of contrast to BMI could lead to better venous opacification, which is why we propose including this factor in the standard MPMCTA protocol.

Concerning the digestive system, the artefacts can easily be explained by enzymatic autolysis; we observed artefacts 
Fig. 8 Prevalence of inhomogeneous vessel opacification (without contrast layering)
Prevalence of an inhomogeneous opacification of the vessels without layer

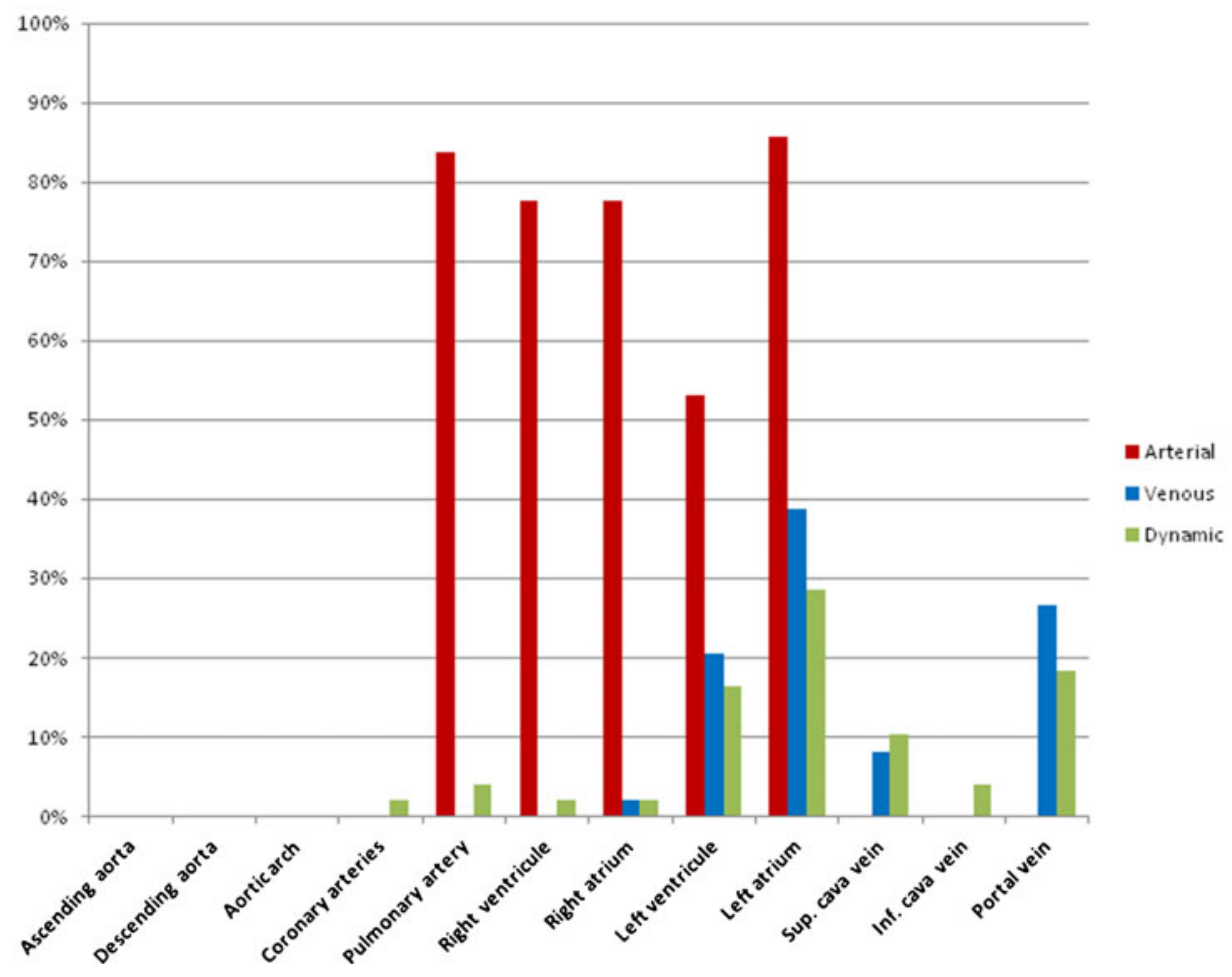

in organs with high enzymatic activity such as the gastric mucosa and the pancreas. In our studies, these artefacts did not depend on the postmortem delay, which was an unexpected finding. In the worst case, an extravasation of the contrast agent into the gastric mucosa can be observed, implying that when toxicological analysis of the gastric content is important, the content should be extracted before contrast agent injection to prevent contamination, or postmortem CT angiography should even be avoided. Because an investigation for intoxication is not an indication for MPMCTA, this characteristic does not appear to be a real limitation. Another problem is the interpretation of intragastric bleeding. This diagnosis should be made only in cases in which a slight enhancement of the mucosa is visible and not in cases where a clear oedema can be observed. Therefore, radiologists and forensic pathologists should handle the diagnosis of intragastric bleeding with care, and the degree of gastrointestinal artefacts should be considered when making such a diagnosis. In addition, diagnosis of a peripancreatic bleeding can be performed only in cases of clear signs of the bleeding on native CT scans (i.e. presence of peripancreatic blood on the native CT scan and extravasation of contrast agent in this exact region) . The simple finding of contrast agent around the head of the pancreas must be interpreted as artefact.

Concerning the vascular system, two types of artefacts can be observed. One is the presence of a layer in the arteries. Contrast layering was seen only in large vessels such as the aortic arch, descending aorta and inferior vena cava. The most important discriminating factor to use in avoiding mistaking such artefacts for a dissection is the interface between the overlying contrast layer and the dependent blood. Angiofil ${ }^{\circledR}$ (a contrast agent consisting of iodized linseed oil) typically forms a horizontal interface with the underlying native blood. True dissection, on the other hand, usually produces a semiluminar interface. The second feature to consider is the aspect of the layer in the different phases. A true dissection flap should be seen in all phases, but an artefactual contrast layering will typically be seen clearly in the arterial phase only, before fading or disappearing in the venous and dynamic phases.

More difficult to interpret is the presence of filling defects in the vascular system. As our study shows, such inhomogeneous opacification without layering appears mostly in the cardiac cavities and the pulmonary arteries. If there is no layer, these artefacts cannot be clearly identified as such unless they change their appearance during the different phases of angiography. However, as the correlation to the autopsy finding showed, in most cases, these artefacts result from huge postmortem clots that occlude the vascular lumen and that can create images identical to those of real vascular occlusion such as pulmonary embolism, even if these artefacts are without correlation to any vital coagulation process. Unfortunately, the presence of the postmortem clots 
Fig. 9 Prevalence of fluid or tissue sampling-related artefacts
Prevalence of artefacts due to sample collection

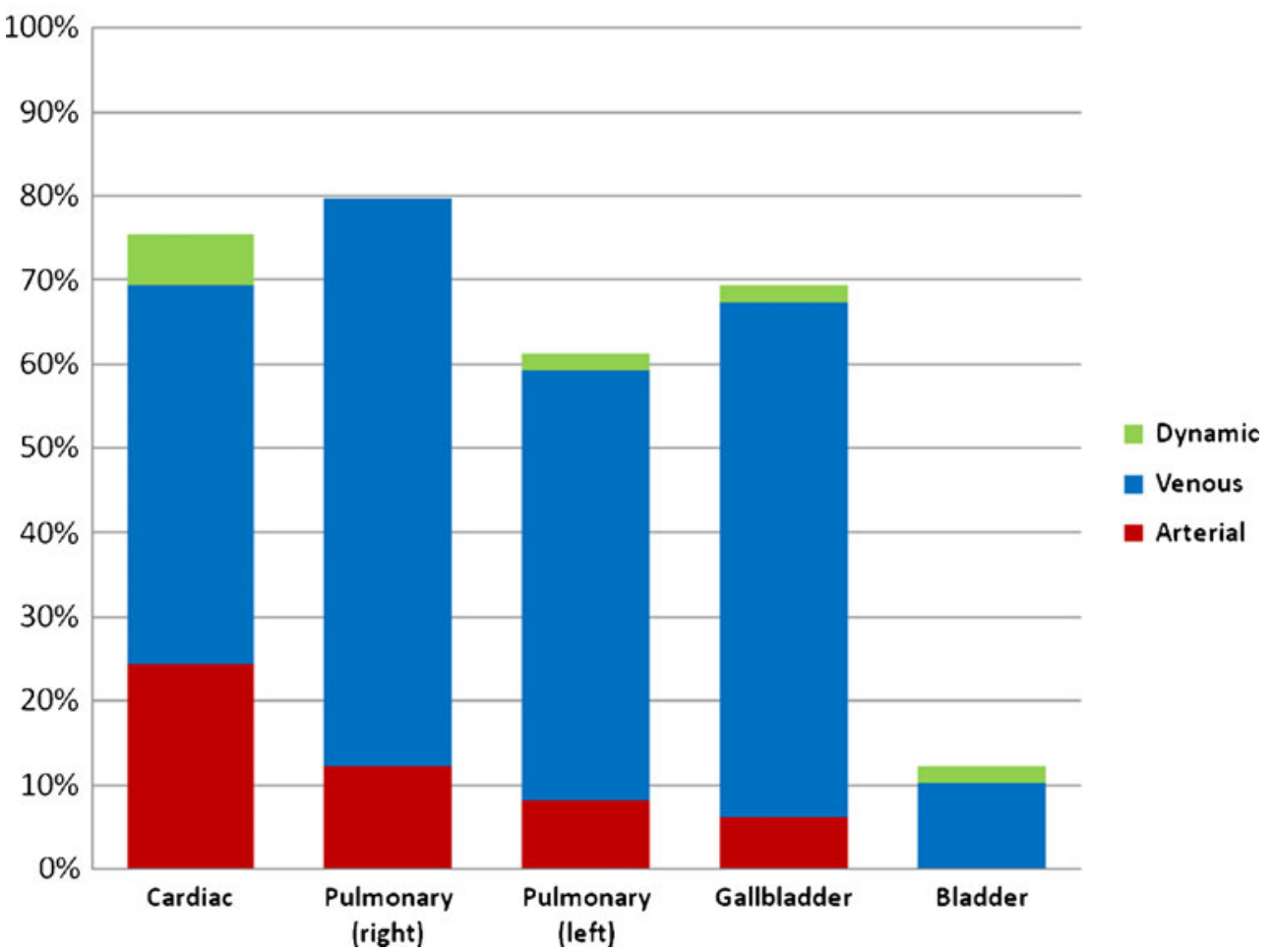

was not always described in the autopsy reports so that an exact correlation of their prevalence and the appearance of the artefact could not be analysed. We could not find a clear difference between artefacts created by postmortem clots and real embolism; thus, the diagnosis of an antemortem pulmonary embolism should remain an autopsy diagnosis and only be suspected but not confirmed after MPMCTA. Nevertheless, in cases where we observed such filling artefacts, we identified them in many different parts of the vascular system and not only in the pulmonary arteries. These artefacts seem to be related to a prolonged phase of agony (or deaths related to other causes than polytrauma). For the practical use, this means that in cases with a long period of agony with a high prevalence of such artefacts, it is not possible to verify with certitude the presence of an antemortem occlusion.

Perihilar renal contrast extravasation at the level of the renal vein, particularly on the right side, was rarely observed (seven cases). This location is the only one where this phenomenon has ever been observed since MPMCTA was introduced at our institution. One possible explanation is a pressure-related rupture induced by our perfusion system. The right renal vein might constitute the point of highest pressure and vulnerability when contrast is injected through the cannulated right femoral vein, which is the side most often cannulated, although this remains to be seen. To differentiate this artefactual contrast extravasation from a perihilar hematoma (resulting from an antemortem lacerated or injured renal vein, for example), the images should be correlated with the initial native CT. If no spontaneous density is present on the native study, extravasation should be considered as an artefact.

Concerning the prevalence of artefacts and the parameters of the examined bodies, we found that the most important factor is the condition of death, especially the phase of agony. Cases with no artefacts are mostly cases of polytrauma and the frequency of artefacts, especially those of the vascular system, increases with a prolonged phase of agony. Therefore, this frequency of artefacts could indicate whether death happened after a long phase of agony or not. Of course, this hypothesis based on these data from our work would require testing in further studies.

Finally, artefacts resulting from CT-guided tissue and fluid sampling before contrast injection were frequently observed. To differentiate pathologic extravasation from sampling artefacts and avoid interpretation errors during autopsy, we thus modified our MPMCTA protocol after completion of the present study. To perform as few sample collections as possible, the forensic pathologist in charge of the case determines their necessity. As a way to facilitate artefact recognition, biopsy needles and needles for the liquid samples should remain in the body after the sample collection during one short native scan to indicate the position of each needle. In this way, a potential artefact observed during autopsy can easily be verified as such. 
Limitations The present study tried to evaluated artefacts which have been observed during the first years of introduction of the MPMCTA into forensic pathology. Our centre was the first one to use this new technique, and therefore, until today no guidelines or recommendations exists how to interpret the obtained images. Starting with this major limitation, we tried to discover pitfalls and to define artefacts that have been recognised. Obviously, all described artefacts depend therefore on the experience that we have made and had been chosen in a subjective way. Although we tried to establish a complete list of them and we had an independent observer to view the data, we cannot guarantee that other users of the technique would have found more artefacts that are not described here.

In summary, each technique performed on the body, whether by conventional autopsy, histology or other examinations, creates artefacts, but once the artefacts are wellknown and recognized as such, there is less danger of misinterpretation. All artefacts that can be observed using MPMCTA seem to be reproducible and stable in terms of their localization and type, making them more or less easily recognizable. The artefacts fall into three categories: related to postmortem changes, related to the method and iatrogenic artefacts related to the samples collections. An understanding of these artefacts can help avoid misinterpretations during radiological evaluation, and an understanding of their meaning is important for guaranteeing a correct radiological interpretation.

Acknowledgments This study was financially supported by the Promotion Agency for Innovation of the Swiss Confederation (KTI Nr.10221.1 PFIW-IW) and by the Fondation Leenaards, Lausanne, Switzerland.

Conflicts of interest The authors have no conflicts of interest to disclose.

\section{References}

1. Grabherr S, Djonov V, Yen K et al (2007) Postmortem angiography: review of former and current methods. AJR 188:832-838

2. Schoenmackers J (1960) Technique of postmortem angiography with reference to related methods of postmortem blood vessel demonstration [in German]. Ergebn Allg Pathol Anat 39:53-151

3. Schlesinger JM (1938) An injection plus dissection study of coronary artery occlusions and anastomosis. Am Heart J 15:528-568

4. Dirnhofer R et al (2006) VIRTOPSY: minimally invasive, imaging-guided virtual autopsy. Radiographics 26(5):1305-1333

5. Thali M, Dirnhofer R, Vock P (eds) (2009) The virtopsy approach: $3 \mathrm{D}$ optical and radiological scanning and reconstruction in forensic medicine. CRC, New York

6. Jeffery AJ (2010) The role of computed tomography in adult postmortem examinations: an overview. Diagn Histopathol 16(12):546-551
7. O'Donnell C (2010) An image of sudden death: utility of routine post-mortem computed tomography scanning in medico-legal autopsy practice. Diagn Histopathol 16(12):552-555

8. Weustink AC et al (2009) Minimally invasive autopsy: an alternative to conventional autopsy? Radiology 250(3):897-904

9. Poulsen K, Simonsen J (2007) Computed tomography as a routine in connection with medico-legal autopsies. Forensic Sci Int 171(23):190-197

10. Roberts IS, Benamore RE, Benbow EW, Lee SH, Harris JN, Jackson A, Mallett S, Patankar T, Peebles C, Roobottom C, Traill ZC (2012) Postmortem imaging as an alternative to autopsy in the diagnosis of adult deaths: a validation study. Lancet 379(9811):136-142

11. Saunders $\mathrm{S}$ et al (2010) Post-mortem computed tomography angiography: past, present and future. Forensic Sci Med Pathol 7:271-277

12. Grabherr S, Djonov V, Friess A et al (2006) Postmortem angiography after vascular perfusion with diesel oil and a lipophilic contrast agent. AJR 187:W515-W523

13. Jackowski C, Thali M, Sonnenschein M et al (2005) Virtopsy: postmortem minimally invasive angiography using cross section techniques -implementation and preliminary results. J Forensic Sci 50:1175-1186

14. Grabherr S et al (2008) Two-step postmortem angiography with a modified heart lung machine: preliminary results. AJR 190(2):345-351

15. Ross S, Spendlove D, Bolliger S (2008) Postmortem whole-body CT angiography: evaluation of two contrast media solutions. AJR 190:1380-1389

16. Roberts ISD, Peebles C, Roobottom C, Traill ZC (2011) Diagnosis of coronary artery disease using minimally invasive autopsy: evaluation of a novel method of postmortem coronary CT angiography. Clin Radiol 66(7):645-650

17. Saunders S, Morgan B, Raj V, Robinson C, Rutty G (2011) Targeted postmortem computed tomography cardiac angiography: proof of concept. Int J Leg Med 125:609-616

18. Grabherr S et al (2010) Multi-phase post-mortem CT angiography: development of a standardized protocol. Int J Leg Med 125:791-802

19. Michaud K, Grabherr S, Doenz F, Mangin P (2012) Evaluation of postmortem MDCT and MDCT angiography for the investigation of sudden cardiac death related to atherosclerotic coronary artery disease. Int J Cardiovasc Imaging 28:1807-1822

20. Palmiere C (2012) Detection of hemorrhage source: the diagnostic value of post-mortem CT angiography. Forensic Sci Int 222:33-39. doi:10.1016/j.forsciint.2012.04.031

21. Zerlauth JB, Doenz F, Dominguez A, Palmiere C, Uské A, Meuli R, Grabherr S (2013) Surgical interventions with fatal outcome: utility of multi-phase postmortem CT angiography. Forensic Sci Int 225:32-41

22. Batra P, Bigoni B, Manning J, Aberle DR, Brown K, Hart E, Goldin J (2000) Pitfalls in the diagnosis of thoracic aortic dissection at CT angiography. Radiographics 20(2):309-320

23. Wittram C, Maher MM, Yoo AJ, Kalra MK, Shepard J-A O, McLoud TC (2004) CT angiography of pulmonary embolism: diagnostic criteria and causes of misdiagnosis. Radiographics 24:1219-1238. doi:10.1148/rg.245045008

24. Schneider B, Chevallier C, Dominguez A, Bruguier C, Elandoy C, Mangin P, Grabherr S (2012) The forensic radiographer: a new member in the medico-legal team. Am J Forensic Med Pathol Am J Forensic Med Pathol 33(1):30-36

25. Anonymous (2000) Recommendation no R(99)3 of the committee of ministers to member states on the harmonization of medicolegal autopsy rules. Council of Europe, Committee of Ministers. Adopted by the Committee of Ministers on 2 February 1999 at the 658th meeting of the Ministers' Deputies

26. Brinkmann B (1999) Harmonisation of medico-legal autopsy rules. Int J Legal Med 113(1):1-14 